\title{
Stability Analysis of Networked Control Systems with Random Time Delays and Packet Dropouts Modeled by Markov Chains
}

\author{
Li Qiu, ${ }^{1}$ Fengqi Yao, ${ }^{2}$ and Xiaopin Zhong $^{1}$ \\ ${ }^{1}$ Shenzhen Key Laboratory of Urban Rail Transit, College of Mechatronics and Control Engineering, \\ Shenzhen University, Shenzhen 518060, China \\ ${ }^{2}$ School of Electrical Engineering \& Information, Anhui University of Technology, Maianshan 243000, China \\ Correspondence should be addressed to Xiaopin Zhong; xzhong@szu.edu.cn
}

Received 27 May 2013; Revised 30 July 2013; Accepted 31 July 2013

Academic Editor: Baocang Ding

Copyright (C) $2013 \mathrm{Li}$ Qiu et al. This is an open access article distributed under the Creative Commons Attribution License, which permits unrestricted use, distribution, and reproduction in any medium, provided the original work is properly cited.

\begin{abstract}
This paper investigates the stability analysis problem for a class of discrete-time networked control systems (NCSs) with random time delays and packet dropouts based on unified Markov jump model. The random time delays and packet dropouts existed in feedback communication link are modeled by two independent Markov chains; the resulting closed-loop system is described by a new Markovian jump linear system (MJLS) with Markov delays. Sufficient conditions of the stochastic stability for NCSs is obtained by constructing a novel Lyapunov functional, and the mode-dependent output feedback controller design method is presented based on linear matrix inequality (LMI) technique. A numerical example is given to illustrate the effectiveness of the proposed method.
\end{abstract}

\section{Introduction}

Networked control systems (NCSs) are a type of closedloop systems, in which the control loops are closed through communication networks. Compared with the traditional control systems, the use of the communication networks bring many advantages such as low cost, reduced weight, and simple installation and maintenance as well as high efficiency, flexibility, and reliability. Consequently, NCSs are applied in a broad range such as manufacturing plants, vehicles, aircrafts, spacecrafts, and remote surgery [1]. However, the communication networks in control loops also present some constraints such as time delays and packet dropouts due to limited bandwidth; quantization errors caused by hybrid nature of NCSs; variable sampling or transmission intervals due to multiple nodes; clock asynchronization among local and remote nodes; network security and safety and network security due to shared communication networks $[2,3]$. It is generally known that any of these networked-induced communication imperfections and constraints can degrade closed-loop performance or, even worse, can harm closedloop stability of NCSs. Therefore, it is important to know how these effects influence the stability properties. Recently, some important results of NCSs have been reported in the existing literature for instance, the discussions of packet dropouts [413], time delays [14-24], quantization [25], distributed synchronization [26], communication constraints [27], stability and controller design [28-33], both data quantizations and packet losses [34], both time delays and packet dropouts [35$44]$, and output feedback control problem $[19,45,46]$.

In NCSs, time delays and packet dropouts are two important issues. To study these issues, many efforts have been made for NCSs with time delays [14-24] and packet dropouts [4-13]; for more details review, please refer to the literature therein. However, both time delays and packet dropouts exist in NCSs by the insertion of communication network in the feedback control loop. Xie and Xia [35] studied the robust fault tolerant controller design for NCSs with fast varying delay and packet dropout. Zhang and Yu [36] presented a switched system model to describe the NCSs with both delay and packet dropout, and the state feedback stabilizing controllers are designed by augmenting technique. Yu and Shi [37] addressed the two-mode-dependent state feedback controller design in NCSs with time delays and packet dropouts by augmenting the state variable approach. Dong et al. [38] studied the robust $H_{\infty}$ filtering problem 
for a class of uncertain nonlinear networked systems with both stochastic time-varying communication delays and packet dropouts. Jiang et al. [39] introduced the design of observer-based controller for NCSs with network induced delay and packet dropout. Li et al. [40] considered the observer-based fault detection problem for NCSs with long time delays and packet dropout by modeling the observers system as an uncertain switched system. Li et al. [41] studied the guaranteed const control of NCSs with the S-C packet dropouts and time delays. Wang and Yang [42] considered the problem of $H_{\infty}$ controller design for NCSs with time delay and packet dropout by applying the linear estimationbased time delay and packet dropout compensation method. Liu et al. [43] investigated the receding horizon $H_{\infty}$ control problem for a class NCSs with random delay and packet disordering by using the receding optimization principle. Qiu et al. [44] considered the state feedback control problem of NCS with both time delays and packet dropouts. In [19, $45,46]$, the output feedback control problem of NCSs were investigated. To the best of the authors' knowledge, up to now, little attention has been paid to the study of NCSs with random time delays and packet dropouts based on Markov jump unified model, which motivates our investigation.

In this paper, we address the unified model and stability analysis problem of NCSs with the random time delays and packet dropouts under a Markovian jump linear system (MJLS) framework. The feedback communication link random time delays and packet dropouts are modeled by two independent Markov chains, the resulting closed-loop system is modeled as a new MJLS with Markov delays. Then, we give stability analysis and output feedback controller design which are for discrete-time NCSs with both time delays and packet dropouts by the Lyapunov stability theory and linear matrix inequality method.

Notations. In the sequel, if not explicit, matrices are assumed to have appropriate dimensions. $\mathbb{R}^{n}$ and $\mathbb{R}^{n \times m}$ denote, respectively, the $n$ dimensional Euclidean space and the set of all $n \times m$ real matrices. The notations $A>0$ and $A<0$ are used to denote the positive and negative definite matrix, respectively. $\operatorname{diag}\left(A_{1}, \ldots, A_{n}\right)$ refers to a $n \times n$ diagonal matrix with $A_{i}$ as its $i$ th diagonal entry. $I$ and 0 denote the identity matrix and zero matrix with appropriate dimensions, respectively. The superscript ${ }^{T}$ denotes the transpose for vectors or matrices. $\mathbb{E}[\cdot]$ denotes the mathematical expectation operator. The symbol $*$ denotes blocks that are readily inferred by symmetry.

\section{Problem Description}

The framework of the system over a network medium is depicted in Figure 1. Considering the same assumption in [14], the sensor, the controller, and the actuator are timedriven and are connected over a network medium. Under the assumption, it is known that the controller updates at the instant $k$ will always use the most recent data; otherwise, it will maintain the old data. In the NCS as Figure 1, network

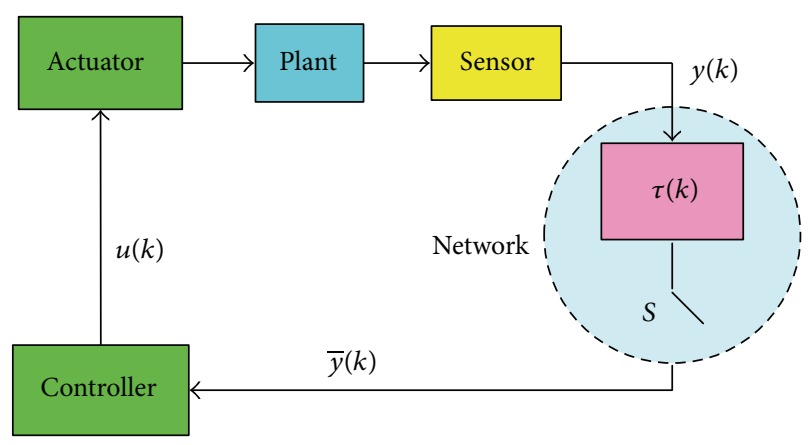

FIGURE 1: The structure of the NCS with random delays and/or packet dropouts.

induced time delays and packet dropouts exist in the feedback communication link.

The discrete-time plant with a time-varying controller is described as

$$
\begin{gathered}
x_{p}(k+1)=A x_{p}(k)+B u(k), \\
y(k)=C x_{p}(k),
\end{gathered}
$$

where $x_{p}(k) \in \mathbb{R}^{n}$ is the system state, $u(k) \in \mathbb{R}^{m}$ is the control input, and $y(k) \in \mathbb{R}^{p}$ is measurable output. $A, B$, and $C$ are known real constant matrices with appropriate dimensions. Random time delays and packet dropouts exist in link from sensor-to-controller (S-C), as shown in Figure 1. Here, $\tau(k)$ represents the bounded random S-C time delays. One way to model the delays $\tau(k)$ is using the finite state Markov chain as shown in [17-19]. The main advantage of the Markov model is that the dependencies between the delays are taken into account since the current time delays in real networks are usually related with the previous delays [17]. In this paper $\tau(k)$ is modeled as a homogeneous Markov chain that take values in $\bar{S}_{2}=\left\{0,1, \ldots, s_{2}\right\}$. $S$ denotes the network switches between the S-C. $\alpha(k)(\alpha(k)=0,1)$ denotes the states of $S$. When $S$ is in state $\alpha(k)=0$, the packet is received successfully and the $\bar{y}(k)=y(k-\tau(k))$. Whereas when $S$ is in state $\alpha(k)=1$, the packet is lost and the switch output is held at the previous value $\bar{y}(k)=\bar{y}(k-1)$. The behavior of the S-C time delays and packet dropouts can be modeled as

$$
\bar{y}(k)=(1-\alpha(k)) y(k-\tau(k))+\alpha(k) \bar{y}(k-1),
$$

where

$$
\alpha(k)= \begin{cases}0, & \text { if } S \text { is closed and the packet is received, } \\ 1, & \text { if } S \text { is open and the packet is lost. }\end{cases}
$$

Considering the mode-dependent output feedback controller:

$$
u(k)=K(\alpha(k), \tau(k)) \bar{y}(k),
$$

where $K(\alpha(k), \tau(k))$ is the output feedback controller gain. 
Let $x(k)=\left[x_{p}(k)^{T} \bar{y}(k-1)^{T}\right]^{T}$ be the augmented state vector. Under the control (4), the closed-loop system of (1) is

$$
\begin{gathered}
x(k+1)=\bar{A}(\alpha(k)) x(k)+\bar{B}(\alpha(k)) H x(k-\tau(k)), \\
x(k)=\varphi(k), \quad k=-\tau_{\max },-\tau_{\max }+1, \ldots, 0,
\end{gathered}
$$

where $\bar{A}(\alpha(k))=\left[\begin{array}{cc}A & \alpha(k) B K(\alpha(k), \tau(k)) \\ 0 & \alpha(k) I\end{array}\right], \bar{B}(\alpha(k))=$ $\left[\begin{array}{c}(1-\alpha(k)) B K(\alpha(k), \tau(k)) C \\ (1-\alpha(k)) C\end{array}\right], H=\left[\begin{array}{ll}I & 0\end{array}\right], \tau_{\max }=\max \{\tau(k)\}$, and $\varphi(k)$ is the initial condition of $x(k)$.

In system (5), $\{\alpha(k), k \in \mathbb{Z}\}$ and $\{\tau(k), k \in \mathbb{Z}\}$ are two independent discrete-time homogeneous Markov chains taking value in a finite set $\bar{S}_{1}=\{0,1\}$ and $\bar{S}_{2}=\left\{0,1, \ldots, s_{2}\right\}$ with transition probabilities:

$$
\begin{gathered}
\operatorname{Pr}\{\alpha(k+1)=j \mid \alpha(k)=i\}=\pi_{i j}, \quad \pi_{i}=\operatorname{Pr}\left(\pi_{0}=i\right), \\
\operatorname{Pr}\{\tau(k+1)=n \mid \tau(k)=m\}=\lambda_{m n}, \\
\lambda_{m}=\operatorname{Pr}\left(\lambda_{0}=m\right),
\end{gathered}
$$

where $\pi_{i j} \geq 0$ and $\lambda_{m n} \geq 0$ for all $i, j \in \bar{S}_{1}, m, n \in \bar{S}_{2}$ and

$$
\sum_{j=0}^{1} \pi_{i j}=1, \quad \sum_{n=0}^{s_{2}} \lambda_{m n}=1 .
$$

For $\alpha(k)=i, i \in \bar{S}_{1}$, when $\alpha(k)$ in mode $i=0$ and $i=1$, the $\alpha(k)$ in (5) take value $\alpha(k)=0$ and $\alpha(k)=$ 1, respectively. $\bar{A}(\alpha(k))$ and $\bar{B}(\alpha(k))$ are known constant matrices of appropriate dimensions.

Remark 1. The closed-loop system (5) is a MJLS with two Markov chains, which describe the behavior of the S-C time delays and packet dropouts, respectively. This enables us to analyze and synthesize such NCSs by applying MJLS theory. Note that modeling the S-C time delays and packet dropouts simultaneously in NCSs based on unified Markov jump model has not been done in the literature.

Definition 2 (see [19]). The system in (5) is stochastically stable if for every finite $x_{0}=x(0)$, initial mode $\alpha_{0}=\alpha(0) \epsilon$ $\bar{S}_{1}$, and $\tau_{0}=\tau(0) \in \bar{S}_{2}$, there exists a finite $\mathscr{W}>0$ such that the following holds:

$$
\mathbb{E}\left\{\sum_{k=0}^{\infty}\|x(k)\|^{2} \mid x_{0}, \alpha_{0}, \tau_{0}\right\}<x_{0}^{T} \mathscr{W} x_{0} .
$$

\section{Main Results}

By applying a new Lyapunov functional, sufficient conditions for the stochastic stability and synthesis of the modedependent output feedback controller design for system (5) will be established in this section.

Theorem 3. For system (5), given random but bounded scalar $\tau(k) \in\left[\begin{array}{ll}\tau_{\min } & \tau_{\max }\end{array}\right]$, if for each mode $i \in \bar{S}_{1}, m \in \bar{S}_{2}$, there exist matrices $P_{i, m}>0, Q_{1}>0, Q_{2}>0, Q_{3}>0, R_{1}>0$, and $R_{2}>0$ such that the following matrix inequalities:

$$
\left[\begin{array}{cccc}
\Xi_{1} & \Xi_{2} & \Xi_{3} & \Xi_{4} \\
* & -\bar{P}_{i, m} & 0 & 0 \\
* & * & -R_{1} & 0 \\
* & * & * & -R_{2}
\end{array}\right]<0,
$$

where

$$
\begin{aligned}
& \Xi_{1}=\left[\begin{array}{cccc}
\Pi_{i, m} & H^{T} R_{1} & 0 & 0 \\
R_{1} H & -Q_{3}-2 R_{1}-2 R_{2} & R_{2} & R_{1}+R_{2} \\
0 & R_{2} & -Q_{2}-R_{2} & 0 \\
0 & R_{1}+R_{2} & 0 & -Q_{1}-R_{1}-R_{2}
\end{array}\right] \text {, } \\
& \Xi_{2}=\left[\begin{array}{llllll}
\bar{P}_{i, m} \bar{A}_{i} & \bar{P}_{i, m} \bar{B}_{i} & 0 & 0
\end{array}\right]^{T}, \\
& \Xi_{3}=\left[\begin{array}{llll}
\tau_{\max } R_{1} H\left(\bar{A}_{i}-I\right) & \tau_{\max } R_{1} H \bar{B}_{i} & 0 & 0
\end{array}\right]^{T} \text {, } \\
& \Xi_{4}=\left[\begin{array}{llll}
\tilde{\tau} R_{2} H\left(\bar{A}_{i}-I\right) & \tilde{\tau} R_{2} H \bar{B}_{i} & 0 & 0
\end{array}\right]^{T}, \\
& \widetilde{\tau}=\tau_{\max }-\tau_{\min }, \\
& \bar{P}_{i, m}=\sum_{j=0}^{1} \sum_{n=0}^{s_{2}} \pi_{i j} \lambda_{m n} P_{j, n}, \\
& \Pi_{i, m}=-P_{i, m}+H^{T}\left(Q_{1}+Q_{2}\right) H \\
& +\left(\tau_{\max }-\tau_{\min }+1\right) H^{T} Q_{3} H-H^{T} R_{1} H, \\
& \bar{A}_{i}=\left[\begin{array}{cc}
A & i B K(i, m) \\
0 & i I
\end{array}\right] \text {, } \\
& \bar{B}_{i}=\left[\begin{array}{c}
(1-i) B K(i, m) C \\
(1-i) C
\end{array}\right] \text {, }
\end{aligned}
$$

and $H$ is defined in (5).

Hold for all $i, j \in \bar{S}_{1}$ and $m, n \in \bar{S}_{2}$; then system (5) is stochastically stable.

Proof. For the closed-loop system (5), stochastic Lyapunov functional is constructed as follows:

$$
\begin{aligned}
V(x(k), \alpha(k), \tau(k)) & =\sum_{\rho=1}^{4} V_{\rho}(x(k), \alpha(k), \tau(k)) \\
& =\sum_{\rho=1}^{4} V_{\rho},
\end{aligned}
$$

where

$$
\begin{gathered}
V_{1}=x(k)^{T} P(\alpha(k), \tau(k)) x(k), \\
V_{2}=\sum_{l=k-\tau_{\max }}^{k-1} x(l)^{T} H^{T} Q_{1} H x(l)+\sum_{l=k-\tau_{\min }}^{k-1} x(l)^{T} H^{T} Q_{2} H x(l), \\
V_{3}=\sum_{\theta=-\tau_{\max }+2}^{-\tau_{\min }+1} \sum_{l=k+\theta-1}^{k-1} x(l)^{T} H^{T} Q_{3} H x(l) \\
+\sum_{l=k-\tau(k)}^{k-1} x(l)^{T} H^{T} Q_{3} H x(l),
\end{gathered}
$$




$$
\begin{array}{r}
V_{4}=\sum_{\theta=-\tau_{\max }+1}^{0} \sum_{l=k+\theta-1}^{k-1} \tau_{\max } \delta(l)^{T} H^{T} R_{1} H \delta(l) \\
+\sum_{\theta=-\tau_{\max }+1}^{-\tau_{\min }} \sum_{l=k+\theta-1}^{k-1}\left(\tau_{\max }-\tau_{\min }\right) \\
\times \delta(l)^{T} H^{T} R_{2} H \delta(l),
\end{array}
$$

and $\delta(l)=x(l+1)-x(l)$. In the following when $\alpha(k)=i$ and $\tau(k)=m$, we will write $P(\alpha(k), \tau(k)), K(\alpha(k), \tau(k)), \bar{A}(\alpha(k))$ and $\bar{B}(\alpha(k))$ as $P_{i, m}, K_{i, m}, \bar{A}_{i}$, and $\bar{B}_{i}$, respectively. We denote:

$$
\begin{aligned}
\Delta V & (x(k), \alpha(k), \tau(k)) \\
& =\sum_{\rho=1}^{4} \Delta V_{\rho} \\
& =\sum_{\rho=1}^{4}\left[V_{\rho}(x(k+1), \alpha(k+1), \tau(k+1) \mid x(k),\right. \\
& \left.\alpha(k), \tau(k))-V_{\rho}(x(k), \alpha(k), \tau(k))\right] .
\end{aligned}
$$

Let $\xi(k)=\left[x(k)^{T}(H x(k-m))^{T}\left(H x\left(k-\tau_{\min }\right)\right)^{T}\right.$ $\left.\left(H x\left(k-\tau_{\max }\right)\right)^{T}\right]^{T}$. Then, along the solution of system (5) we have

$$
\begin{aligned}
\mathbb{E}\left[\Delta V_{1}\right]= & x(k+1)^{T}\left[\sum_{j=0}^{1} \sum_{n=0}^{s_{2}} \pi_{i j} \lambda_{m n} P_{j, n}\right] \\
& \times x(k+1)-x^{T}(k) P_{i, m} x(k) \\
= & \xi^{T}(k)\left[\begin{array}{c}
\bar{A}_{i}^{T} \\
\bar{B}_{i}^{T} \\
0 \\
0
\end{array}\right] \bar{P}_{i, m}\left[\begin{array}{llll}
\bar{A}_{i} & \bar{B}_{i} & 0 & 0
\end{array}\right] \xi(k) \\
& -x^{T}(k) P_{i, m} x(k),
\end{aligned}
$$

where $\bar{P}_{i, m}$ is defined in Theorem 3 .

We have

$$
\begin{aligned}
\mathbb{E}\left[\Delta V_{2}\right]= & x^{T}(k) H^{T}\left(Q_{1}+Q_{2}\right) H x(k) \\
& -x^{T}\left(k-\tau_{\max }\right) H^{T} Q_{1} H x\left(k-\tau_{\max }\right) \\
& -x^{T}\left(k-\tau_{\min }\right) H^{T} Q_{2} H x\left(k-\tau_{\min }\right), \\
\mathbb{E}\left[\Delta V_{3}\right]= & \left(\tau_{\max }-\tau_{\min }+1\right) x(k)^{T} H^{T} Q_{3} H x(k) \\
& -\sum_{\theta=k-\tau_{\max }+1}^{k-\tau_{\min }} x(l)^{T} H^{T} Q_{3} H x(l) \\
& +\left(\sum_{l=k-n+1}^{k-1}-\sum_{l=k-m+1}^{k-1}\right) x(l)^{T} H^{T} Q_{3} H x(l) \\
& -x(k-m)^{T} H^{T} Q_{3} H x(k-m) .
\end{aligned}
$$

Note that

$$
\begin{aligned}
\sum_{l=k-n+1}^{k-1} x^{T}(l) H^{T} Q_{3} H x(l) \\
=\left[\sum_{l=k-\tau_{\min }+1}^{k-1}+\sum_{l=k-n+1}^{k-\tau_{\min }}\right] x^{T}(l) H^{T} Q_{3} H x(l) \\
\leq\left[\sum_{l=k-m+1}^{k-1}+\sum_{l=k-\tau_{\max }+1}^{k-\tau_{\min }}\right] x^{T}(l) H^{T} Q_{3} H x(l) .
\end{aligned}
$$

By combining (16) and (17), we have

$$
\begin{aligned}
\mathbb{E}\left[\Delta V_{3}\right] \leq & \left(\tau_{\max }-\tau_{\min }+1\right) x(k)^{T} H^{T} Q_{3} H x(k) \\
& -x(k-m)^{T} H^{T} Q_{3} H x(k-m), \\
\mathbb{E}\left[\Delta V_{4}\right]= & \tau_{\max }^{2} \delta^{T}(k) H^{T} R_{1} H \delta(k) \\
& -\sum_{l=k-\tau_{\max }}^{k-1} \tau_{\max } \delta^{T}(l) H^{T} R_{1} H \delta(l) \\
& +\left(\tau_{\max }-\tau_{\min }\right)^{2} \delta^{T}(k) H^{T} R_{2} H \delta(k) \\
& -\sum_{l=k-\tau_{\min }-1}\left(\tau_{\max }-\tau_{\min }\right) \delta^{T}(l) H^{T} R_{2} H \delta(l) .
\end{aligned}
$$

By Jensen's inequality, we can get

$$
\begin{aligned}
\sum_{l=k-\tau_{\max }}^{k-1} \tau_{\max } \delta^{T}(l) H^{T} R_{1} H \delta(l) & \left(\sum_{l=k-\tau_{\max }}^{k-\tau_{k}-1}+\sum_{l=k-\tau_{k}}^{k-1}\right)\left(\tau_{\max }-\tau_{k}+\tau_{k}\right) \\
= & \times \delta^{T}(l) H^{T} R_{1} H \delta(l) \\
\geq & \left(\tau_{\max }-\tau_{k}\right) \sum_{l=k-\tau_{\max }}^{k-\tau_{k}-1} \delta^{T}(l) H^{T} R_{1} H \delta(l) \\
& +\tau_{k} \sum_{l=k-\tau_{k}}^{k-1} \delta^{T}(l) H^{T} R_{1} H \delta(l) \\
\geq & \left(\sum_{l=k-\tau_{\max }}^{k-\tau_{k}-1} \delta(l)\right)^{T} H^{T} R_{1} H\left(\sum_{l=k-\tau_{\max }}^{k-\tau_{k}-1} \delta(l)\right) \\
& +\left(\sum_{l=k-\tau_{k}}^{k-1} \delta(l)\right)^{T} H^{T} R_{1} H\left(\sum_{l=k-\tau_{k}}^{k-1} \delta(l)\right)
\end{aligned}
$$




$$
\begin{aligned}
& \geq\left(x\left(k-\tau_{\max }\right)-x\left(k-\tau_{k}\right)\right)^{T} H^{T} R_{1} H \\
& \quad \times\left(x\left(k-\tau_{\max }\right)-x\left(k-\tau_{k}\right)\right) \\
& \quad+\left(x\left(k-\tau_{k}\right)-x(k)\right)^{T} H^{T} R_{1} H\left(x\left(k-\tau_{k}\right)-x(k)\right) \\
& \geq \xi(k)^{T}\left\{\left[\begin{array}{cccc}
H^{T} R_{1} H & -H^{T} R_{1} & 0 & 0 \\
-R_{1} H & 2 R_{1} & 0 & -R_{1} \\
0 & 0 & 0 & 0 \\
0 & -R_{1} & 0 & R_{1}
\end{array}\right]\right\} \xi(k) .
\end{aligned}
$$

Similarly, we have

$$
\begin{aligned}
& \sum_{l=k-\tau_{\max }}^{k-\tau_{\min }-1}\left(\tau_{\max }-\tau_{\min }\right) \delta^{T}(l) H^{T} R_{2} H \delta(l) \\
& =\left(\sum_{l=k-\tau_{\max }}^{k-\tau_{k}-1}+\sum_{l=k-\tau_{k}}^{k-\tau_{\min }-1}\right) \\
& \quad \times\left(\tau_{\max }-\tau_{k}+\tau_{k}-\tau_{\min }\right) \delta^{T}(l) H^{T} R_{2} H \delta(l) \\
& \geq \xi(k)^{T}\left\{\left[\begin{array}{cccc}
0 & 0 & 0 & 0 \\
0 & 2 R_{2} & -R_{2} & -R_{2} \\
0 & -R_{2} & R_{2} & 0 \\
0 & -R_{2} & 0 & R_{2}
\end{array}\right]\right\} \xi(k) .
\end{aligned}
$$

$$
\begin{aligned}
& \mathbb{E}[\Delta V] \leq \xi^{T}(k)\left\{\begin{array}{cccc}
\Pi_{i, m} & H^{T} R_{1} & 0 & 0 \\
R_{1} H & -Q_{3}-2 R_{1}-2 R_{2} & R_{2} & R_{1}+R_{2} \\
0 & R_{2} & -Q_{2}-R_{2} & 0 \\
0 & R_{1}+R_{2} & 0 & -Q_{1}-R_{1}-R_{2}
\end{array}\right] \\
& +\left[\begin{array}{c}
\bar{A}_{i}^{T} \\
\bar{B}_{i}^{T} \\
0 \\
0
\end{array}\right] \bar{P}_{i, m}\left[\begin{array}{llll}
\bar{A}_{i} & \bar{B}_{i} & 0 & 0
\end{array}\right]+\left[\begin{array}{c}
\tau_{\max }\left(H\left(\bar{A}_{i}-I\right)\right)^{T} \\
\tau_{\max }\left(H \bar{B}_{i}\right)^{T} \\
0 \\
0
\end{array}\right] R_{1}\left[\tau_{\max } H\left(\bar{A}_{i}-I\right) \tau_{\max } H \bar{B}_{i} \quad 0 \quad 0\right]
\end{aligned}
$$

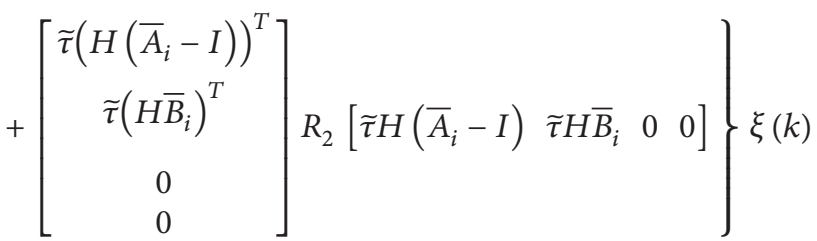

$$
\begin{aligned}
& =\xi^{T}(k) \Theta_{i, m} \xi(k),
\end{aligned}
$$

where $\Pi_{i, m}$ and $\tilde{\tau}$ are defined in Theorem 3 .

By Schur complement and from (9), we have $\Theta_{i, m}<0$. Therefore,

$$
\mathbb{E}[\Delta V] \leq-\boldsymbol{\lambda}_{\min }\left(-\Theta_{i, m}\right) \xi(k)^{T} \xi(k) \leq-\boldsymbol{\eta} x(k)^{T} x(k),
$$

where $\lambda_{\min }\left(-\Theta_{i, m}\right)$ denotes the minimal eigenvalue of $-\Theta_{i, m}$
By combining (19), (20), and (21), we have

$$
\begin{aligned}
& \mathbb{E}\left[\Delta V_{4}\right] \leq \xi(k)^{T}\left\{\left[\begin{array}{c}
\tau_{\max }\left(H\left(\bar{A}_{i}-I\right)\right)^{T} \\
\tau_{\max }\left(H \bar{B}_{i}\right)^{T} \\
0 \\
0
\end{array}\right]\right. \\
& \times R_{1}\left[\tau_{\max } H\left(\bar{A}_{i}-I\right) \tau_{\max } H \bar{B}_{i} \quad 0 \quad 0\right] \\
& +\left[\begin{array}{c}
\tilde{\tau}\left(H\left(\bar{A}_{i}-I\right)\right)^{T} \\
\tilde{\tau}\left(H \bar{B}_{i}\right)^{T} \\
0 \\
0
\end{array}\right] \\
& \times R_{2}\left[\begin{array}{llll}
\tilde{\tau} H\left(\bar{A}_{i}-I\right) & \tilde{\tau} H \bar{B}_{i} & 0 & 0
\end{array}\right] \\
& +\left[\begin{array}{cccc}
-H^{T} R_{1} H & H^{T} R_{1} & 0 & 0 \\
R_{1} H & -2 R_{1} & 0 & R_{1} \\
0 & 0 & 0 & 0 \\
0 & R_{1} & 0 & -R_{1}
\end{array}\right] \\
& \left.+\left[\begin{array}{cccc}
0 & 0 & 0 & 0 \\
0 & -2 R_{2} & R_{2} & R_{2} \\
0 & R_{2} & -R_{2} & 0 \\
0 & R_{2} & 0 & -R_{2}
\end{array}\right]\right\} \xi(k),
\end{aligned}
$$

where $\widetilde{\tau}$ is defined in Theorem 3 .

By combining (14), (15), (18), and (22), we have

and $\boldsymbol{\eta}=\inf \left\{\lambda_{\min }\left(-\Theta_{i, m}\right)\right\}$. From (24), it is seen that for any $t>0$

$$
\begin{aligned}
\mathbb{E} & {[V(x(k+1), \alpha(k+1), \tau(k+1))] } \\
& -\mathbb{E}[V(\varphi, \alpha(0), \tau(0))] \leq-\eta \sum_{k=0}^{t} E\left[x(k)^{T} x(k)\right] .
\end{aligned}
$$


Furthermore

$$
\sum_{k=0}^{t} \mathbb{E}\left[x(k)^{T} x(k)\right] \leq \frac{1}{\boldsymbol{\eta}} \mathbb{E}[V(\varphi, \alpha(0), \tau(0))] .
$$

By taking limit as $t \rightarrow \infty$, we have

$$
\sum_{k=0}^{\infty} \mathbb{E}\left[x(k)^{T} x(k)\right] \leq \frac{1}{\eta} \mathbb{E}[V(\varphi, \alpha(0), \tau(0))]<\infty .
$$

According to Definition 2, the closed-loop system (5) is stochastically stable. This completes the proof.

Theorem 3 gives the sufficient conditions for the stochastic stability of system (5). However, it should be noted that the conditions (9) are no more LMI conditions. To handle this, the equivalent LMI conditions are given in Theorem 4 by Cone Complementarity Linearization (CCL) algorithm.
Theorem 4. Consider system (5) with random but bounded $\operatorname{scalar} \tau(k) \in\left[\begin{array}{ll}\tau_{\min } & \tau_{\max }\end{array}\right]$. There exists an output feedback controller (4) such the resulting closed-loop system is stochastically stable if for each mode $i \in \bar{S}_{1}, m \in \bar{S}_{2}$, there exist matrices $P_{i, m}>0, X_{i, m}>0, Q_{1}>0, Q_{2}>0, Q_{3}>0, R_{1}>0, R_{2}>0$, $U_{1}>0, U_{2}>0$, and $K_{i, m}$ such that

$$
\begin{gathered}
{\left[\begin{array}{cccc}
\Xi_{1} & \widehat{\Xi}_{2} & \widehat{\Xi}_{3} & \widehat{\Xi}_{4} \\
* & -X_{j, n} & 0 & 0 \\
* & * & -U_{1} & 0 \\
* & * & * & -U_{2}
\end{array}\right]<0,} \\
P_{i, m} X_{i, m}=I, \quad R_{1} U_{1}=I, \quad R_{2} U_{2}=I,
\end{gathered}
$$

where

$$
\begin{aligned}
& \widehat{\Xi}_{2}=\left[\begin{array}{lllll}
\mathscr{L}_{i, m} \bar{A}_{i} & \mathscr{L}_{i, m} \bar{B}_{i} & 0 & 0
\end{array}\right]^{T}, \\
& \widehat{\Xi}_{3}=\left[\begin{array}{llll}
\tau_{\max } H\left(\bar{A}_{i}-I\right) & \tau_{\max } H \bar{B}_{i} & 0 & 0
\end{array}\right]^{T} \text {, }
\end{aligned}
$$

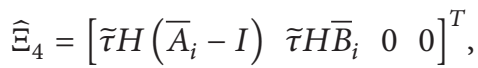

$$
\begin{aligned}
& X_{j, n}=\operatorname{diag}\left\{X_{0,0}, X_{0,1}, \ldots, X_{0, s_{2}}, X_{1,0}, X_{1,1}, \ldots, X_{1, s_{2}}\right\} \text {, }
\end{aligned}
$$

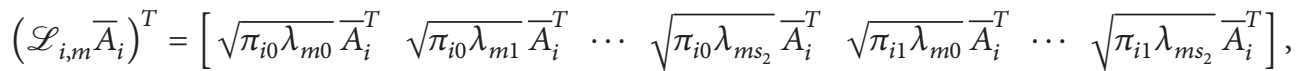

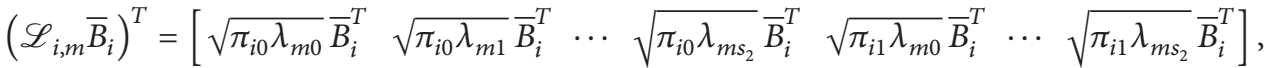

$$
\begin{aligned}
& \bar{A}_{i}=\left[\begin{array}{cc}
A & 0 \\
0 & i I
\end{array}\right]+\left[\begin{array}{c}
i B \\
0
\end{array}\right] K_{i, m}\left[\begin{array}{ll}
0 & I
\end{array}\right], \\
& \bar{A}_{i}-I=\left[\begin{array}{cc}
A-I & 0 \\
0 & (i-1) I
\end{array}\right]+\left[\begin{array}{c}
i B \\
0
\end{array}\right] K_{i, m}\left[\begin{array}{ll}
0 & I
\end{array}\right] \text {, } \\
& \bar{B}_{i}=\left[\begin{array}{c}
0 \\
(1-i) C
\end{array}\right]+\left[\begin{array}{c}
(1-i) B \\
0
\end{array}\right] K_{i, m} C \text {, } \\
& H\left(\bar{A}_{i}-I\right)=\left[\begin{array}{ll}
A-I & 0
\end{array}\right]+i B K_{i, m}\left[\begin{array}{ll}
0 & I
\end{array}\right], \\
& H \bar{B}_{i}=(1-i) B K_{i, m} C .
\end{aligned}
$$

$\Xi_{1}$ and $\tilde{\tau}$ are defined in Theorem 3. Moreover, if (28) (29) have solutions, the controller gain is given by $K_{i, m}$.

Proof. By Schur complement, (28) is equivalent to

$$
\left[\begin{array}{cccc}
\Xi_{1} & \widehat{\Xi}_{2} & \widehat{\Xi}_{3} & \widehat{\Xi}_{4} \\
* & -P_{j, n}^{-1} & 0 & 0 \\
* & * & -R_{1}^{-1} & 0 \\
* & * & * & -R_{2}^{-1}
\end{array}\right]<0 .
$$

Let $P_{j, n}^{-1}=X_{j, n}, R_{1}^{-1}=U_{1}$, and $R_{2}^{-1}=U_{2}$; we can obtain (28), (29). This completes the proof.

The conditions state in Theorem 4 are a set of LMIs with some matrix inverse constraints. Although they are nonconvex, which prevents us from solving them using the existing convex optimization tool, we can use the con complementary linearization to algorithm transform this problem into the nonlinear minimization problem with LMI constraints as follows:

$$
\begin{aligned}
\min \operatorname{Trace} & \left(\sum_{s=1}^{2} R_{s} U_{s}+\sum_{i=0}^{1} \sum_{m=0}^{s_{2}} P_{i, m} X_{i, m}\right) \\
\operatorname{s} \cdot \mathrm{t}\{ & \left\{\begin{array}{l}
\text { (i) } \operatorname{LMI}(28) \\
\text { (ii) }\left[\begin{array}{cc}
R_{s} & I \\
I & U_{s}
\end{array}\right]>0, \quad s \in\{1,2\}, \\
\text { (iii) }\left[\begin{array}{cc}
P_{i, m} & I \\
I & X_{i, m}
\end{array}\right]>0, \quad i \in \bar{S}_{1}, \quad m \in \bar{S}_{2}
\end{array}\right.
\end{aligned}
$$


The above nonlinear minimization problem can be solved by an iterative algorithm presented in the following.

Algorithm 5. Step 1. Find a feasible solution satisfying LMIs (i), (ii), and (iii) in (32); set as $\left(R_{1}^{0}, U_{1}^{0}, R_{2}^{0}, U_{2}^{0}, P_{i, m}^{0}, X_{i, m}^{0}, K^{0}\right)$ and $k=0$.

Step 2. Solve the following LMI optimization problem for variables $\left(R_{1}, U_{1}, R_{2}, U_{2}, P_{i, m}, X_{i, m}, K\right)$. Minimize trace $\left\{\sum_{s=1}^{2}\left(R_{s}^{k} U_{s}+R_{s} U_{s}^{k}\right)+\sum_{i=0}^{1} \sum_{m=0}^{s_{2}}\left(P_{i, m}^{k} X_{i, m}+P_{i, m} X_{i, m}^{k}\right)\right\}$, subject to LMIs (32). Set $R_{1}^{k+1}=R_{1}, U_{1}^{k+1}=U_{1}, R_{2}^{k+1}=R_{2}, U_{2}^{k+1}=U_{2}$, $P_{i, m}^{k+1}=P_{i, m}, X_{i, m}^{k+1}=X_{i, m}$, and $K^{k+1}=K$.

Step 3. If (31) is satisfied, then exit the iteration. If (31) is not satisfied, let $k=k+1$, and then return to Step 2 .

\section{Numerical Example}

To illustrate the effectiveness of the proposed method, we apply the results in Section 3 to a classical angular positioning system [43] in Figure 2, where $\theta$ is the angular position of the antenna, $\theta_{r}$ is the angular position of the moving object, and the angular velocity of the antenna $\dot{\theta}$ is measurable. The control problem is to use the input voltage to the motor to rotate the antenna so that it always points in the direction of a moving object in the plant. The output feedback controller is designed for the following values of the matrices $A, B$, and C:

$$
\begin{gathered}
A=\left[\begin{array}{cc}
1 & 0.0995 \\
0 & 0.99
\end{array}\right], \\
B=\left[\begin{array}{ll}
0.0039 \\
0.0783
\end{array}\right], \\
C=\left[\begin{array}{cc}
1.4 & 0.8 \\
-0.2 & 0.4
\end{array}\right] .
\end{gathered}
$$

The stochastic jumping parameter $\alpha(k) \in\{0,1\}$ and the random delays involved in system $(5)$ are $\tau(k) \in\{0,1,2\}$; the transition probability matrices $\pi$ and $\lambda$ are taken by

$$
\pi=\left[\begin{array}{cc}
0.4 & 0.6 \\
0.55 & 0.45
\end{array}\right], \quad \lambda=\left[\begin{array}{ccc}
0.36 & 0.54 & 0.1 \\
0.26 & 0.52 & 0.22 \\
0.18 & 0.62 & 0.2
\end{array}\right] \text {. }
$$

Figures 3 and 4 show part of the simulation of the stochastic jumping parameter $\alpha(k)$ and S-C delay $\tau(k)$ governed by their corresponding transition probability matrices, respectively.

The initial value $x(0)=\left[\begin{array}{ll}-0.4 & 0.6\end{array}\right]^{T}$. By Theorem 4, we can obtain the gain matrices $K_{i, m}$ of controller (4) which are constructed as

$$
\begin{aligned}
& K_{0,0}=\left[\begin{array}{ll}
-1.1356 & -1.6672
\end{array}\right], \\
& K_{0,1}=\left[\begin{array}{ll}
-0.2870 & -0.4926
\end{array}\right], \\
& K_{0,2}=\left[\begin{array}{ll}
-0.2898 & -0.4909
\end{array}\right],
\end{aligned}
$$

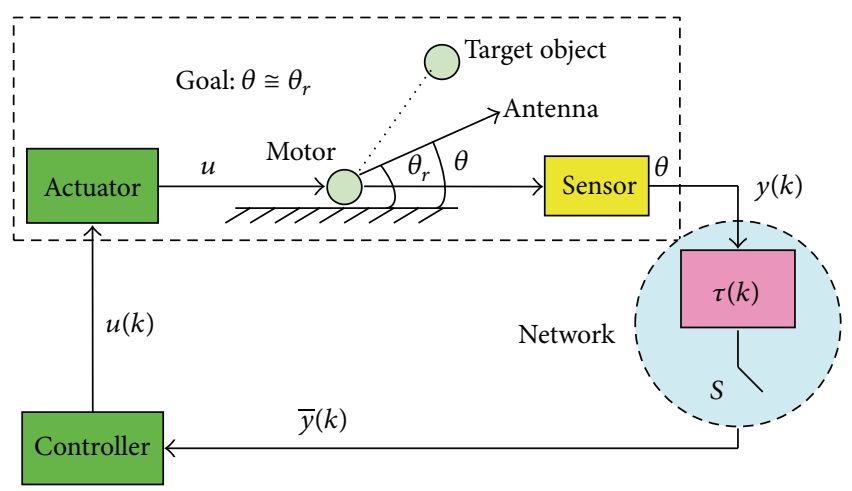

FIgURE 2: The angular positioning system.

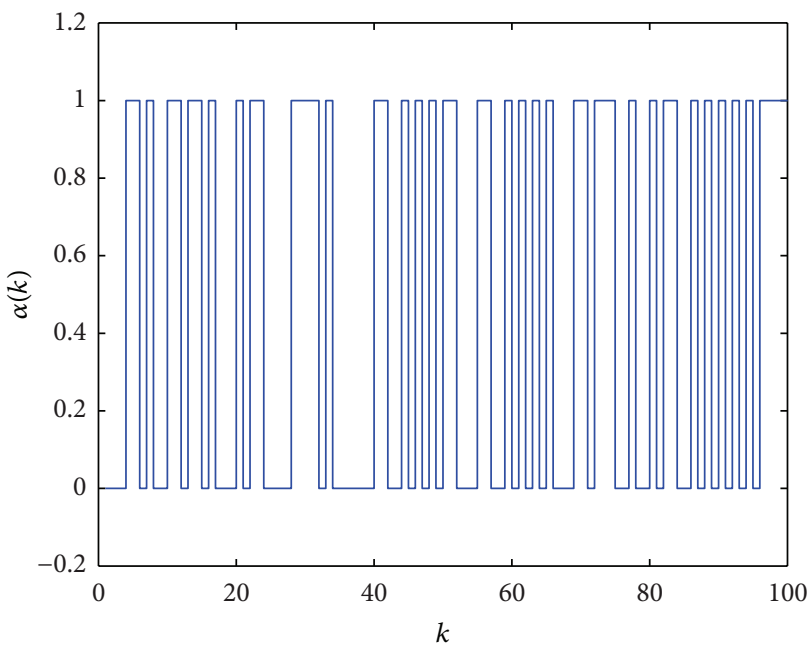

FIGURE 3: Values of $\alpha(k)$.

$$
\begin{aligned}
& K_{1,0}=\left[\begin{array}{ll}
-0.2886 & -0.4900
\end{array}\right], \\
& K_{1,1}=\left[\begin{array}{ll}
-0.2857 & -0.4909
\end{array}\right], \\
& K_{1,2}=\left[\begin{array}{ll}
-0.2829 & -0.4805
\end{array}\right] .
\end{aligned}
$$

The state trajectories and the delay output trajectories are shown in Figures 5 and 6, where four curves represent state trajectories and the delay output trajectories under the controller gains $K_{i, m}$. Figures 5 and 6 indicate that system (5) is stochastically stable. In contrast with the proposed method, the controller gain $K_{\mathrm{dlqr}}$ of a standard linear-quadratic regulator for nominal discrete-time systems designed by MATLAB command dlqr is

$$
K_{\mathrm{dlqr}}=\left[\begin{array}{ll}
0.9332 & 1.6804
\end{array}\right] .
$$

The eigenvalues of $A+B K^{\mathrm{lqr}}$ are 1.1686 and 0.9567 . Hence, $K^{\mathrm{lqr}}$ cannot stabilize the system in this case. The proposed controller works much better for networked control system than the contrastive dlqr method. 


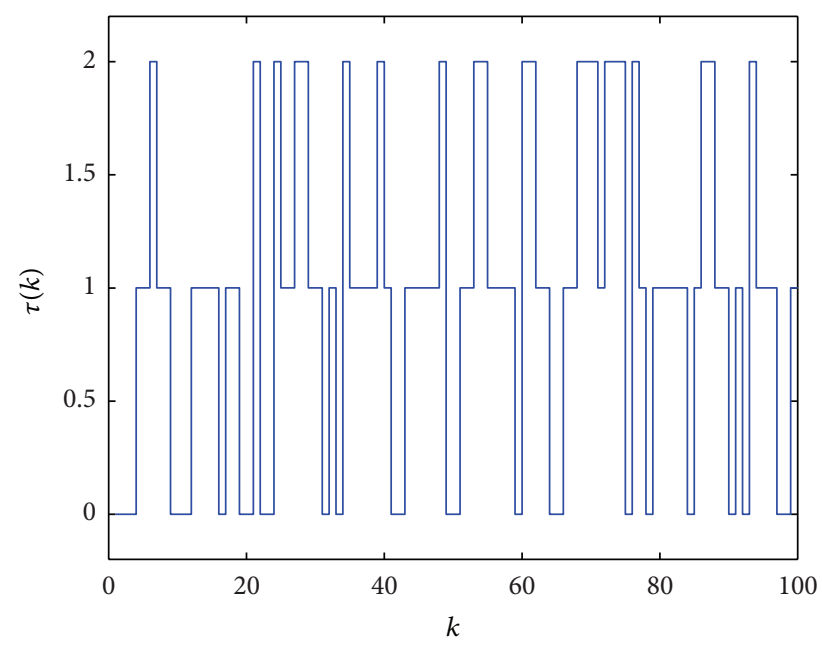

FIgURE 4: S-C random delays $\tau(k)$.

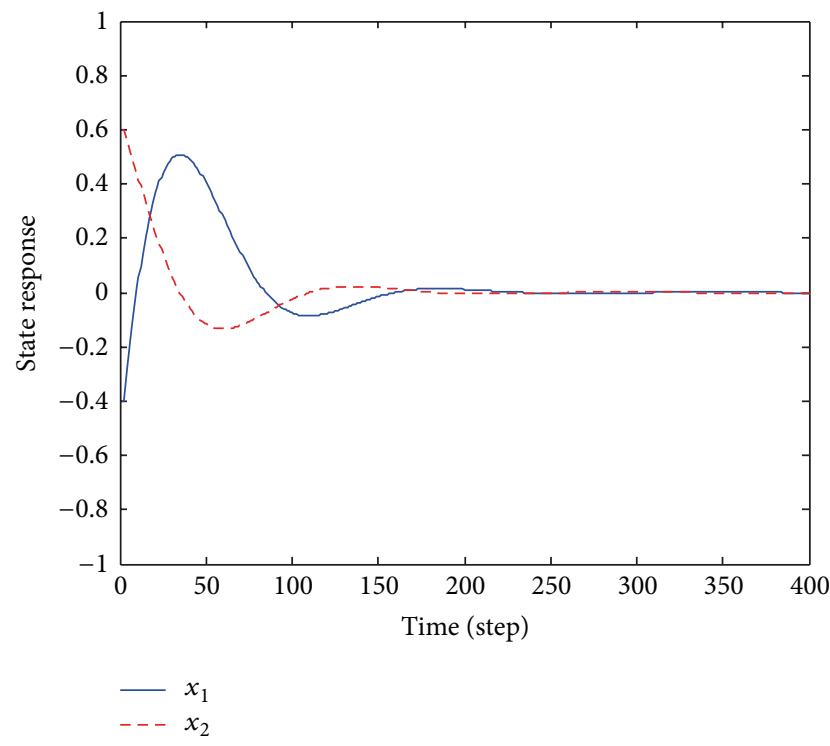

Figure 5: State trajectories under $K_{i, m}$.

\section{Conclusion}

The stability analysis problem for NCSs with random time delays and packet dropouts is investigated in this paper. The random time delays and packet dropouts existed in feedback communication link are modeled by two independent Markov chains. Then the resulting closed-loop system is modeled as a MJLS with Markov delays. Sufficient conditions on stochastic stability and stabilization are obtained by the Lyapunov stability theory and LMI method. The CCL algorithm is employed to obtain the mode-dependent output feedback controller. Finally, an example is presented to illustrate the effectiveness of the approach. Although the NCSs with random time delays and packet dropouts on only sensor to controller link are considered in this paper, the method of unified modeling and the Lyapunov functional constructing can be extended to the NCSs with the random

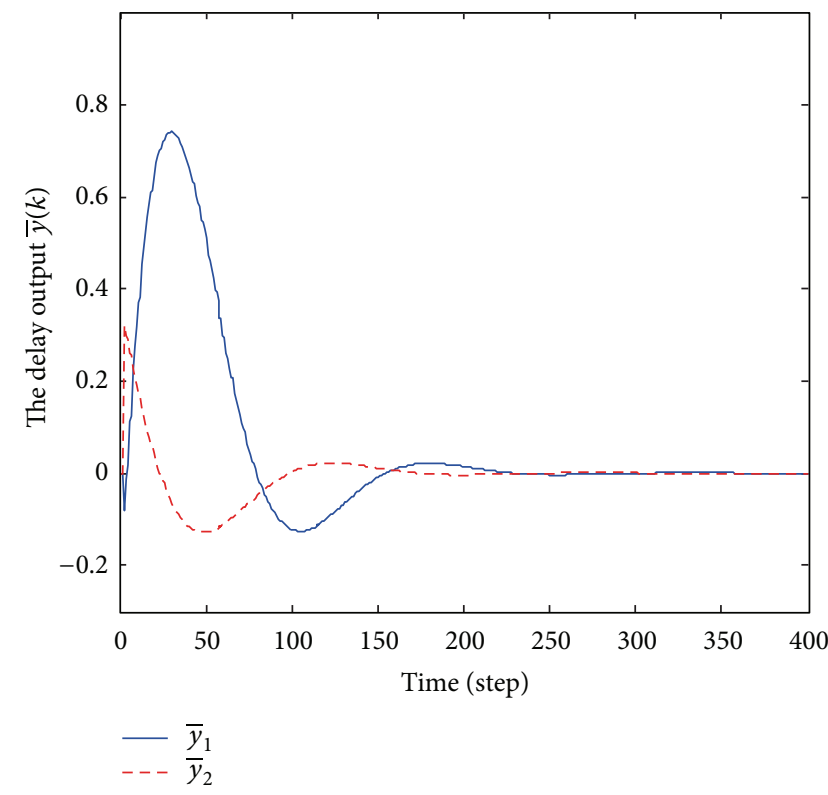

FIGURE 6: The trajectories of delay output $\bar{y}(k)$.

time delays and packet dropouts existing in both the sensor to controller and controller to actuator.

\section{Acknowledgments}

The research is supported by the National Natural Science Foundation of China (Grant no. 61203184) and the Natural Science Foundation of SZU (Grant no. 201207).

\section{References}

[1] J. P. Hespanha, P. Naghshtabrizi, and Y. Xu, "A survey of recent results in networked control systems," Proceedings of the IEEE, vol. 95, no. 1, pp. 138-172, 2007.

[2] M. C. F. Donkers, W. P. M. H. Heemels, N. van de Wouw, and L. Hetel, "Stability analysis of networked control systems using a switched linear systems approach," IEEE Transactions on Automatic Control, vol. 56, no. 9, pp. 2101-2115, 2011.

[3] L. X. Zhang, H. J. Gao, and O. Kaynak, "Network-induced constraints in networked control systems-a survey," IEEE Transactions on Automatic Control, vol. 9, no. 1, pp. 403-416, 2013.

[4] J. Xiong and J. Lam, "Stabilization of linear systems over networks with bounded packet loss," Automatica, vol. 43, no. 1, pp. 80-87, 2007.

[5] Z. Wang, F. Yang, D. W. C. Ho, and X. Liu, "Robust $H_{\infty}$ control for networked systems with random packet losses," IEEE Transactions on Systems, Man, and Cybernetics B, vol. 37, no. 4, pp. 916-924, 2007.

[6] W.-A. Zhang and L. Yu, "Output feedback stabilization of networked control systems with packet dropouts," IEEE Transactions on Automatic Control, vol. 52, no. 9, pp. 1705-1710, 2007.

[7] T. Jia, Y. Niu, and X. Wang, " $H_{\infty}$ control for networked systems with data packet dropout," International Journal of Control, Automation and Systems, vol. 8, no. 2, pp. 198-203, 2010. 
[8] J. Wu and T. Chen, "Design of networked control systems with packet dropouts," IEEE Transactions on Automatic Control, vol. 52, no. 7, pp. 1314-1319, 2007.

[9] B. Xue, S. Li, and Q. Zhu, "Moving horizon state estimation for networked control system with multiple packet dropouts," IEEE Transactions on Automatic Control, vol. 57, no. 9, pp. 2360-2366, 2012.

[10] Q. Lu, L. Zhang, M. Basin, and H. Tian, "Analysis and synthesis for networked control systems with uncertain rate of packet losses," Journal of the Franklin Institute, vol. 349, no. 7, pp. 25002514, 2012.

[11] B. Ding, "Stabilization of linear systems over networks with bounded packet loss and its use in model predictive control," Automatica, vol. 47, no. 11, pp. 2526-2533, 2011.

[12] D. E. Quevedo and D. Nešić, "Robust stability of packetized predictive control of nonlinear systems with disturbances and Markovian packet losses," Automatica, vol. 48, no. 8, pp. 18031811, 2012.

[13] B. Ding and L. Xie, "Stabilization of linear systems over networks with double-sided, finite bounded, arbitrary packet loss," in Proceedings of the IEEE International Conference on Control and Automation (ICCA '09), pp. 920-925, December 2009.

[14] E. Tian and D. Yue, "A new state feedback $H_{\infty}$ control of networked control systems with time-varying network conditions," Journal of the Franklin Institute, vol. 349, no. 3, pp. 891-914, 2012.

[15] D. Yue, E. Tian, and Q.-L. Han, "A delay system method for designing event-triggered controllers of networked control systems," IEEE Transactions on Automatic Control, vol. 58, no. 2, pp. 475-481, 2013.

[16] M. Azadegan, M. T. H. Beheshti, and B. Tavassoli, "Robust output feedback control of networked control systems with random delay modeled by Markov chain," in Proceedings of the 20th Iranian Conference on Electrical Engineering, pp. 587-592, Tehran, Iran, 2012.

[17] J. Nilsson, Real-Time Control Systems with Delays, Lund Institute of Technology, Lund, Sweden, 1998.

[18] L. Xiao, A. Hassibi, and J. P. How, "Control with random communication delays via a discrete-time jump system approach," in Proceedings of the 2000 American Control Conference, pp. 3545-3550, June 2000.

[19] Y. Shi and B. Yu, "Output feedback stabilization of networked control systems with random delays modeled by Markov chains," IEEE Transactions on Automatic Control, vol. 54, no. 7, pp. 1668-1674, 2009.

[20] M. B. G. Cloosterman, N. van de Wouw, W. P. M. H. Heemels, and $\mathrm{H}$. Nijmeijer, "Stability of networked control systems with uncertain time-varying delays," IEEE Transactions on Automatic Control, vol. 54, no. 7, pp. 1575-1579, 2009.

[21] L. Qiu, B. Xu, and S. Li, "Guaranteed cost control for discretetime networked control systems with random Markov delays," Journal of Systems Engineering and Electronics, vol. 22, no. 4, pp. 661-671, 2011.

[22] E. C. Martins and F. G. Jota, "Design of networked control systems with explicit compensation for time-delay variations," IEEE Transactions on Systems, Man and Cybernetics C, vol. 40, no. 3, pp. 308-318, 2010.

[23] I. Pan, S. Das, and A. Gupta, "Tuning of an optimal fuzzy PID controller with stochastic algorithms for networked control systems with random time delay," ISA Transactions, vol. 50, no. 1, pp. 28-36, 2011.
[24] L. Qiu, B. Xu, and S. Li, " $H_{2} / H_{\infty}$ control of networked control system with random time delays," Science China. Information Sciences, vol. 54, no. 12, pp. 2615-2630, 2011.

[25] Y. Ishido, K. Takaba, and D. E. Quevedo, "Stability analysis of networked control systems subject to packet-dropouts and finite-level quantization," Systems \& Control Letters, vol. 60, no. 5, pp. 325-332, 2011.

[26] Y. Tang, H. J. Gao, W. Zou, and J. Kurths, "Distributed synchronization in networks of agent systems with nonlinearities and random switchings," IEEE Transactions on Systems, Man, and Cybernetics B, vol. 43, pp. 358-370, 2013.

[27] Z.-H. Guan, C.-Y. Chen, G. Feng, and T. Li, “Optimal tracking performance limitation of networked control systems with limited bandwidth and additive colored white Gaussian noise," IEEE Transactions on Circuits and Systems I, vol. 60, no. 1, pp. 189-198, 2013.

[28] J. Yu, L. Wang, and M. Yu, "Switched system approach to stabilization of networked control systems," International Journal of Robust and Nonlinear Control, vol. 21, no. 17, pp. 1925-1946, 2011.

[29] T. Suzuki, M. Kono, N. Takahashi, and O. Sato, "Controllability and stabilizability of a networked control system with periodic communication constraints," Systems \& Control Letters, vol. 60, no. 12, pp. 977-984, 2011.

[30] D. Wu, J. Wu, S. Chen, and J. Chu, "Stability of networked control systems with polytopic uncertainty and buffer constraint," IEEE Transactions on Automatic Control, vol. 55, no. 5, pp. 12021208, 2010.

[31] B. Zhu and K. Xia, "Design of subsidence monitoring system based on wireless sensor networks," in Proceedings of the 6th International Conference on Wireless Communications, Networking and Mobile Computing (WiCOM '10), pp. 1-4, September 2010.

[32] Y. Xia, L. Li, G.-P. Liu, and P. Shi, " $H_{\infty}$ predictive control of networked control systems," International Journal of Control, vol. 84, no. 6, pp. 1080-1097, 2011.

[33] P. Wang, C. Han, and B. Ding, "Stability of discrete-time networked control systems and its extension for robust $H_{\infty}$ control," International Journal of Systems Science, vol. 44, no. 2, pp. 275-288, 2013.

[34] X. Tang and B. Ding, "Model predictive control of linear systems over networks with data quantizations and packet losses," Automatica, vol. 49, no. 5, pp. 1333-1339, 2013.

[35] N. Xie and B. Xia, "Robust fault tolerant controller design for uncertain networked control systems with fast-varying delay and packet-dropout," in Proceedings of the 6th International Conference on Wireless Communications, Networking and Mobile Computing (WiCOM '10), pp. 1-4, September 2010.

[36] W.-A. Zhang and L. Yu, "Modelling and control of networked control systems with both network-induced delay and packetdropout," Automatica, vol. 44, no. 12, pp. 3206-3210, 2008.

[37] B. Yu and Y. Shi, "State feedback stabilization of networked control systems with random time delays and packet dropout," in Proceedings of the ASME Dynamic Systems and Control Conference (DSCC '08), pp. 1-7, October 2008.

[38] H. Dong, Z. Wang, and H. Gao, "Robust $H_{\infty}$ filtering for a class of nonlinear networked systems with multiple stochastic communication delays and packet dropouts," IEEE Transactions on Signal Processing, vol. 58, no. 4, pp. 1957-1966, 2010.

[39] C. Jiang, D. X. Zou, and Q. L. Zhang, “Observer-based control for networked control systems with network induced delay and 
packet dropout," in Proceedings of the 8th World Congress on Intelligent Control and Automation (WCICA '10), pp. 4376-4379, July 2010.

[40] X. Li, X. Wu, Z. Xu, and C. Huang, "Fault detection observer design for networked control system with long time-delays and data packet dropout," Journal of Systems Engineering and Electronics, vol. 21, no. 5, pp. 877-882, 2010.

[41] S. B. Li, Y. Q. Wang, F. Xia et al., "Guaranteed cost control of networked guaranteed cost control of networked control systems with time-delays and packet losses," International Journal of Wavelets, Multiresolution and Information Processing, vol. 4, no. 4, pp. 691-7066, 2006.

[42] Y.-L. Wang and G.-H. Yang, "Time delay and packet dropout compensation for networked control systems: a linear estimation method," International Journal of Control, vol. 83, no. 1, pp. 115-124, 2010.

[43] A. Liu, L. Yu, and W. Zhang, "One-step receding horizon $H_{\infty}$ control for networked control systems with random delay and packet disordering," ISA Transactions, vol. 50, no. 1, pp. 44-52, 2011.

[44] L. Qiu, Q. Luo, F. Gong, S. B. Li, and B. G. Xu, "Stability and stabilization of networked control systems with random time delays and packet dropouts," Journal of the Franklin Institute, vol. 350, no. 7, pp. 1886-1907, 2013.

[45] Y. Sun and Y. Huo, "Robust static output feedback control for networked control system with random time delay," in Proceedings of the 2nd International Conference on Advanced Computer Control (ICACC '10), pp. 547-551, March 2010.

[46] C. Peng, Y. Tian, and D. Yue, "Output feedback control of discrete-time systems in networked environments," IEEE Transactions on Systems, Man, and Cybernetics A, vol. 41, no. 1, pp. 185-190, 2011. 


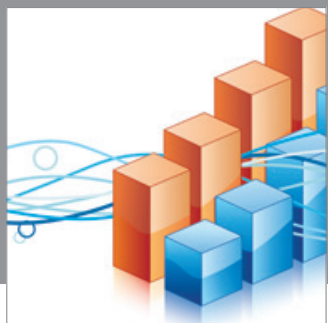

Advances in

Operations Research

mansans

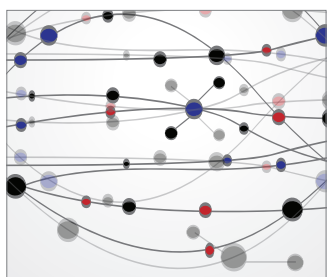

The Scientific World Journal
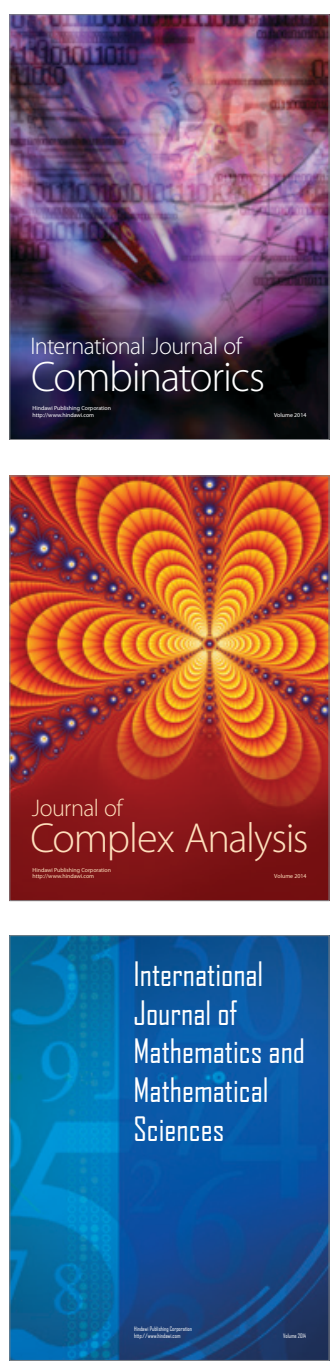
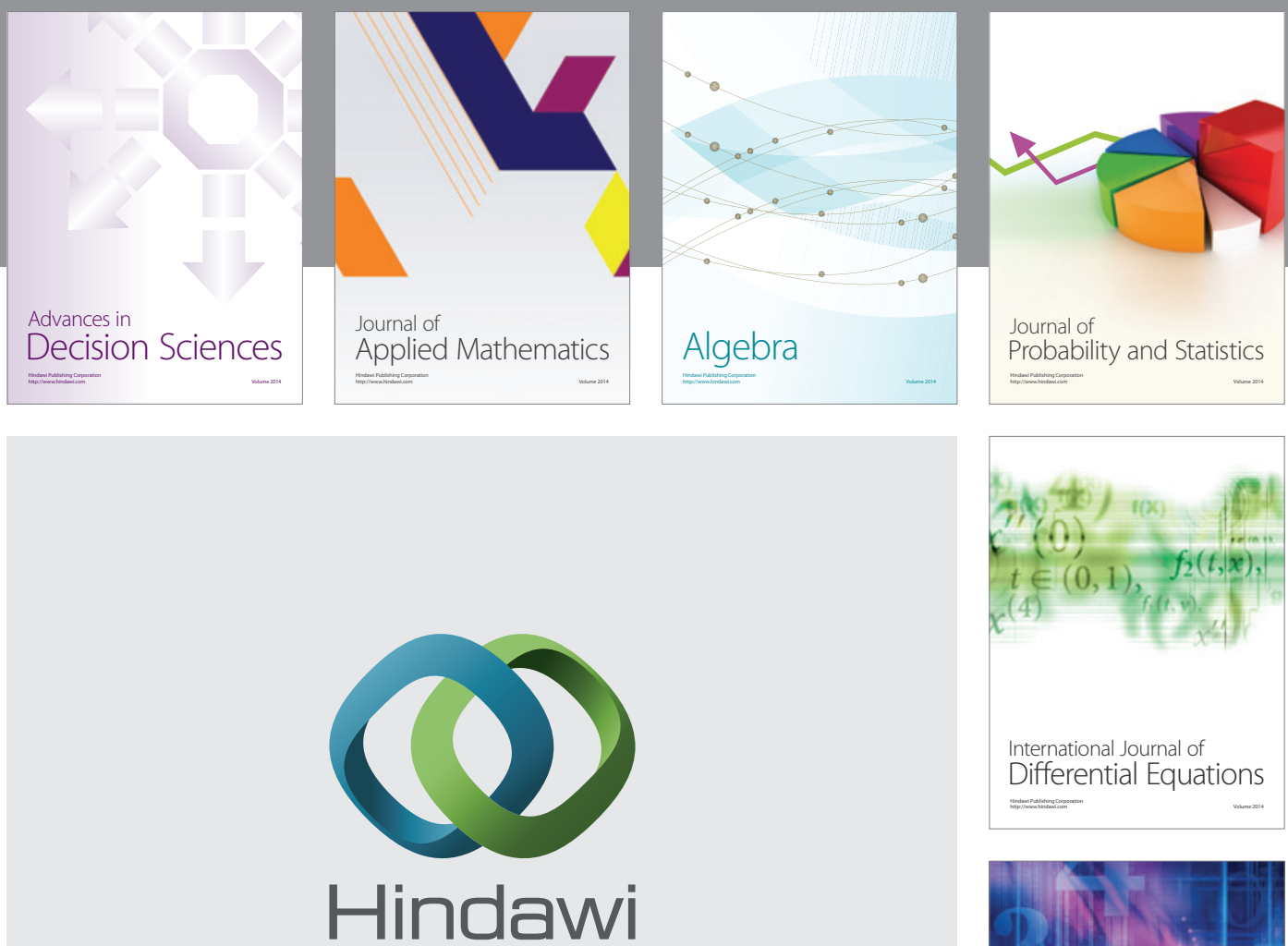

Submit your manuscripts at http://www.hindawi.com
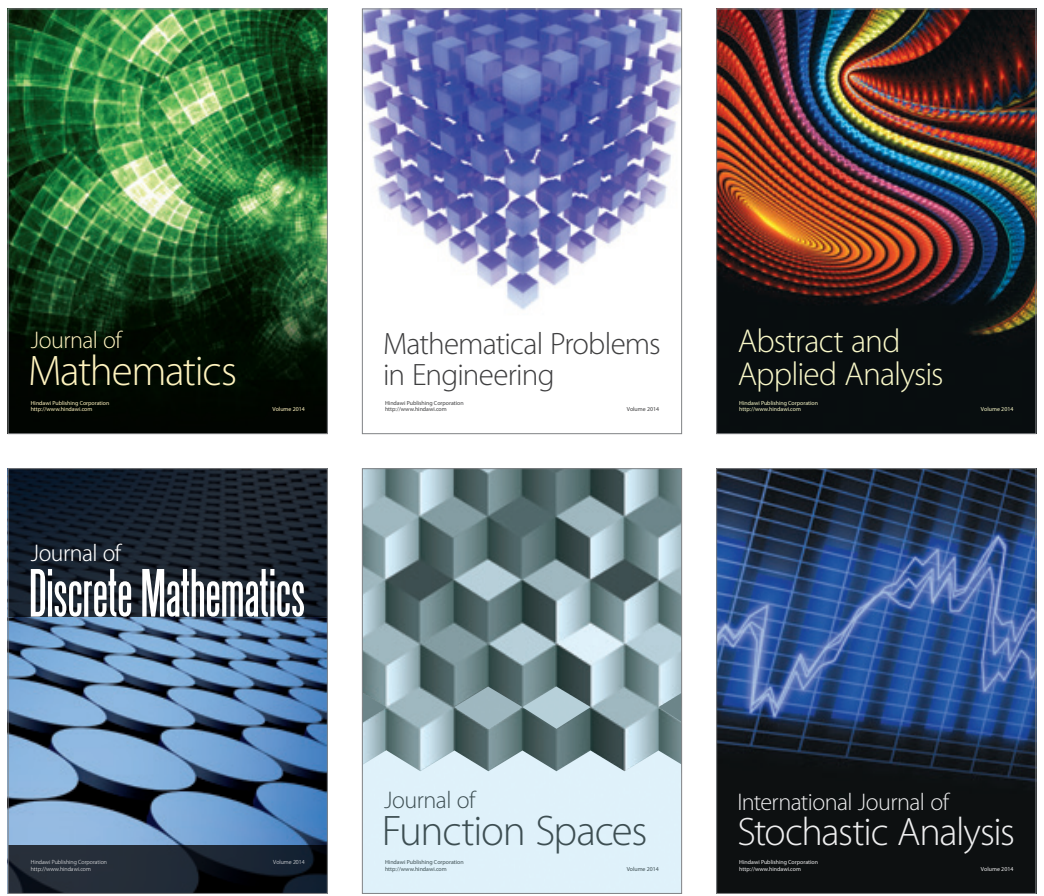

Journal of

Function Spaces

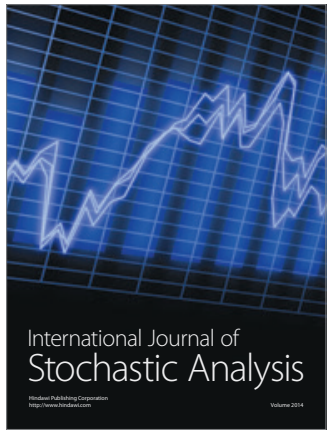

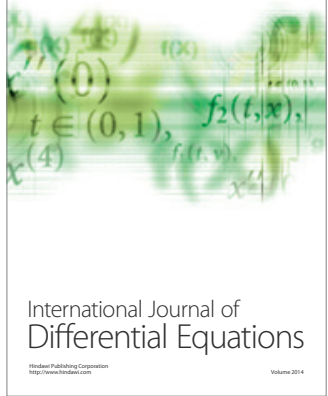
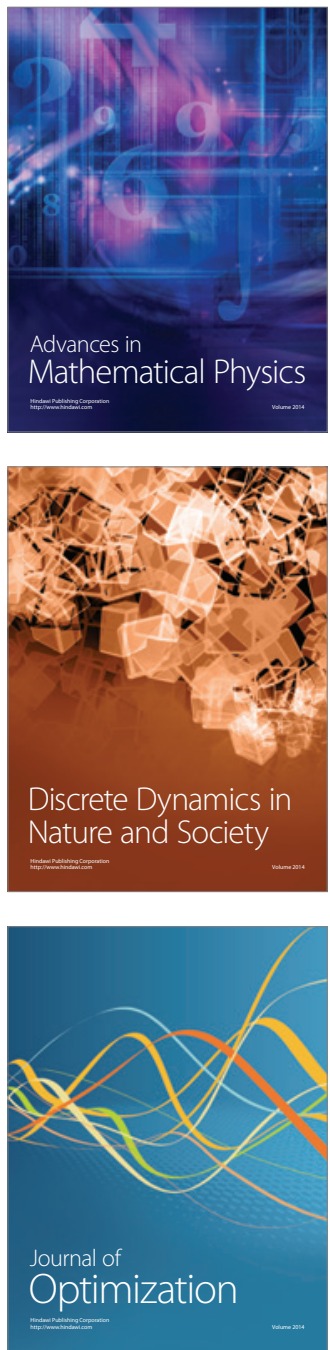